\title{
The Promise of Intergenerational Choir for Improving Psychosocial and Cognitive Health for Those with Dementia: The Voices in Motion Project
}

\author{
Nicholas Tamburri, Michaella Trites, Debra J. Sheets, Andre P. Smith, Stuart W. S. MacDonald* \\ University of Victoria \\ cole.ct25@gmail.com \& mtrites@uvic.ca
}

\begin{abstract}
Rates of dementia continue to increase along with life expectancy. As neither dementia's cause nor its cure is well understood from the perspective of medical science, further investigations of complementary lifestyle and non-pharmaceutical interventions are imperative. Although arts-based therapies have been explored selectively, the significance of these interventions for persons with dementia $(\mathrm{PwD})$ remains undervalued in both the general population and scientific literature. This study aims to examine one promising lifestyle intervention, the effect of intergenerational choir participation, on psychosocial and cognitive function for PwD. Participants $(\mathrm{n}=32)$, in partnership with their family caregivers and local high school students, participated in an intergenerational choir for as many as three choir seasons spanning up to 18 months of follow-up. Participants underwent an expansive assessment of psychosocial, physiological, and cognitive function every four to six weeks as part of an intensive repeated measures design. Here, the potential benefits of choir for PwD were explored in relation to change for select cognitive (Mini-Mental State Examination: MMSE; Trail Making Task A: TMT-A; Word Recall) and psychosocial (Patient Health Questionnaire: PHQ-9) indicators. Multilevel modelling was used to index initial levels (at baseline) and change (spanning up to eight follow-up assessments) in function for measures of global cognition, executive functioning, episodic memory, and depressive symptoms. Notably, no significant declines were observed for MMSE or TMT-A tasks. As expected, episodic memory function continued to decline, with a significant lessening of depressive symptoms and signs observed for the PHQ-9. These results suggest that despite the progressive nature of underlying neuropathology for dementia subtypes like Alzheimer's Disease, preservation of select cognitive functions as well as mitigation of psychosocial comorbidities (depressive symptoms) is possible through participation in an intergenerational choir.

Keywords: dementia; psychosocial function; cognitive function; intergenerational choir; social engagement
\end{abstract}

\section{Introduction}

A A prevailing societal belief is that cognitive and health-related declines are an inevitable part of the ageing process. Although some decline is normal, it is important to emphasize that chronological age itself is not a mechanism that can explain individual vitality or disease risk

${ }^{*}$ C. Tamburri and M. Trites contributed equally to this work, and would like to sincerely thank the amazing contributions of our choral conductor, Erica Phare-Bergh, as well as the equally amazing Voices in Motion research participants. The Voices in Motion Project was supported by grants from the Alzheimer Society of Canada and the Pacific Alzheimer Research Foundation to D.J. Sheets, S.W.S. MacDonald, \& A.P. Smith. S. MacDonald is also supported by the Royal Society of Canada College of New Scholars, Artists and Scientists. 
Notwithstanding, age remains a strong risk factor for several diseases, with dementia among the most notable examples. The incidence of dementia is on the rise, with recent estimates suggesting around 50 million individuals worldwide living with a form of dementia (WHO, 2019). This number is projected to increase to around 152 million cases globally by the year 2050. At present, 500,000 Canadians are living with dementia, a number projected to increase to 1.1 million cases by 2031 (Dudgeon, 2010). This rising prevalence of dementia is mainly attributable to the baby boomer population exceeding 65 years of age, as well as increases in average life expectancy. From a societal perspective, most would argue that there is a social responsibility to ensure proper care for older adults. It is vital to ensure not only that proper infrastructure is available, but also that we are actively pursuing and promoting healthy ageing. There is currently no cure for dementia; thus, alongside continuing biomedical research, lifestyle interventions aimed at promoting health for persons with dementia $(\mathrm{PwD})$ are critical. In keeping with these objectives, this article overviews the Voices in Motion Choir project, an arts-based intervention study focused on the promotion of health and wellbeing for persons with dementia and their caregivers.

\section{Dementia: Context for the Study}

Dementia is not a specific disease per se, but rather an umbrella term that encompasses a collection of symptoms (e.g., impaired memory function, mood changes, language difficulties) due to underlying changes in brain structure and function. There are varying forms and etiologies of dementia, but the most common subtype is Alzheimer's Disease (AD) (Baird \& Samson, 2015). Diagnostic criteria for AD include impairment of memory as well as one other cognitive domain (e.g., language, executive function) that consequently impairs social or occupational functioning (American Psychiatric Association, 2013; Baird \& Samson, 2015). A probabilistic classification of $\mathrm{AD}$ must also be characterized by an insidious onset as well as the gradual progression of impairment (American Psychiatric Association, 2013).

This progression of AD typically advances in a series of distinct clinical stages. Preliminary brain and behavioural changes occur during the preclinical stage of $\mathrm{AD}$, putatively referred to as amnestic mild cognitive impairment (aMCI). The earliest brain changes occur in the entorhinal cortex, a neurological structure connected to the hippocampus that is known to be essential for the storage and retrieval of memories (Braak \& Braak, 1991). These affected regions begin to atrophy and eventually cause memory impairment. Neural atrophy moves outward to the cerebral cortex, impairing not simply memory but other cognitive functions (e.g., executive function, attention, processing speed, general cognition) as well. These additional cognitive changes, coupled with the advancing impairment of activities of daily living (ADLs), mark the transition from aMCI to mild AD (Petersen, 2004). As the disease progresses, common (but not universal) symptoms alongside memory impairment include confusion, mood and personality changes, increased anxiety, language difficulties, difficulty recognizing friends and family, restlessness, repetitive behaviour, perceptual-motor problems, and agitation/irritation (Cummings, 2012). Each of these symptoms shares a close relationship with commensurate changes that are occurring in the brain. During the more severe stages of $\mathrm{AD}$, when considerable neural tissue has been atrophied, individuals with $\mathrm{AD}$ become more dependent upon others for care and maintenance and lose a majority of their autonomy as a consequence. Common observances during these more advanced stages include increasing depressive affect and social isolation, as well as diminished quality of life (Blackburn \& Bradshaw 2014; Cummings, 2012). 


\section{Stigma and Social Isolation}

Significant impairments in social functioning are a direct consequence of the neurological changes of AD. Especially in the later stages of the disease, an individual's capacity for social engagement may be severely truncated due to the AD-related changes in cognition and behavior; namely, $\mathrm{PwD}$ often withdraw from their social spaces and become socially isolated (Hsiao, Chang, \& Gean, 2018). Positive social relationships are strongly linked and positively associated with beneficial outcomes, including improved mental health as well as reduced morbidity and all-cause mortality (Holt-Lunstad, Smith, \& Layton, 2010). In fact, the recently released Harvard Study of Adult Development suggests that social relationships are critical to maintaining health and longevity and that sustaining quality social relationships throughout the lifespan is a significant contributor to late-life health (as cited in Mineo, 2017).

Although the opportunity or capacity to engage socially may be affected by the disease, individuals with dementia benefit from and enjoy relationships and interactions with others. Therefore, the social isolation associated with dementia may be explained better by the impacts of stigma (Behuniak, 2011). Older adults with dementia report that one of the biggest challenges of dementia is the stigma that comes with diagnosis. Stigma perpetuates negative stereotypes about individuals with AD and leads to sensations of "otherness" and social exclusion (Behuniak, 2011; Harris \& Caporella, 2014). In a society where ageism is common, those with dementia experience double jeopardy resulting from the cumulative impact of ageism and dementia stigma (Cohen, 2001; Palmore, 1999).

Research being conducted in the field of arts-based (non-pharmacological) interventions is striving to break down barriers put in place by stigma and bring individuals with and without dementia together as a single community with shared interests. To be sure, numerous social-therapeutic interventions are being examined using a myriad of mediums to bring people together (painting, poetry, dance, etc.), and the present study (Voices in Motion) explores the prospective therapeutic benefits of music, particularly social singing (choir), for improving the psychosocial and cognitive health of those with dementia. Music, indeed, is the shorthand of emotion, and people well into the latter stages of $\mathrm{AD}$ can still engage joyfully in its transcript.

\section{Harnessing the Power of Music for Those with Dementia}

Studies investigating the relationship between music and the brain are relatively nascent, especially when compared to other fields of scientific inquiry. Indeed, music is a grand unifier for social relationships, provoking both our creativity and emotionality, while simultaneously stimulating socialization. This makes music particularly effective as a catalyst for facilitating social therapeutic interventions. Many creativity-based therapeutic interventions yield anecdotal stories and observations of people experiencing joy and merriment who otherwise seemed apathetic, or individuals who have been sedentary for long periods of time finding the urge to tap feet or even stand and clap in response to musical stimuli (Glazner \& Kaplan, 2018). The veracity of these claims has been substantiated in recent research studies demonstrating that various social-cognitive interventions can lead to significant improvements in mood, well-being, and quality of life, with modest gains even observed for cognitive function as the impact of various dementia-related comorbidities are mitigated (Blackburn \& Bradshaw, 2014; Chang et al., 2015).

Music-based interventions have shown promising findings in this respect. Although dementias such as Alzheimer's Disease are not reversible, and music cannot repair the structural damage caused by $\mathrm{AD}$, some dementia-related symptoms (e.g., cognitive impairment) may be attenuated through improving affect, decreasing depressive symptoms and signs, and increasing engagement of neurological resources. To this end, it may be possible to protect remaining function in behavioural and cognitive domains through music. Logically, one can assume that music should facilitate 
socialization and potentially bolster mood and positive affect. Indeed, the success of most social therapeutic interventions is in part contingent not so much on what creative muse the group delights in - only that they all enjoy and wish to engage in it (Baird \& Samson, 2009).

What is less intuitive, however, and what has been gradually accruing more research support, is the seeming ability for individuals with AD to remember old songs, to sing and engage with them more lucidly, and to retain such abilities late into the disease progression. To be sure, music is not an "island of preservation" in the brain, unaffected by the atrophy and implications of disease progression; however, areas of the brain associated with specific parts of musical memory (e.g., procedural memory, emotional memory) may atrophy much later in the disease process, leading to the relative sparing of musical memory within AD (Clark \& Warren, 2015; Jacobsen et al., 2015; Peck et al., 2016). It is well established that memory function, as a broad term, can be categorized into simpler domains that are accomplished in part by differentially activated neural pathways (Squire, 2009). For example, episodic memories - memories that consist of recalling personal details and life experiences - are specifically impaired in $\mathrm{AD}$, leading one to the conclusion that the hippocampus and broader entorhinal cortex are predominantly responsible for the ability to recall such memories. And yet, the procedural memory system that contributes to the training and automaticity of learning skills, such as playing an instrument or riding a bike, is relatively spared throughout the progression of AD (Jacobsen et al., 2015). Not surprisingly, procedural memory is functionally contingent upon regions of the brain that undergo late or modest atrophy in $\mathrm{AD}$.

Another memory system that aids in understanding music's interaction with the brain is semantic memory: the ability to recall facts or general knowledge about the world that is accumulated over the lifespan. In the context of music, semantic memory refers to our factual understanding of musical structure and form, or to the associative or emotional concepts provoked by music that is not linked to the retrieval of specific personal experience (Baird \& Samson, 2009). Semantic musical-memory sparing in $\mathrm{AD}$ has been documented in several publications reporting that individuals with $\mathrm{AD}$ are still able to recognize familiar songs and even lyrics despite episodic recollection deficits (Baird \& Samson, 2015; Jacobsen et al., 2015). One potential explanation for this sparing relates to regions of the brain that serve to consolidate and store long-term memory for music. Regions such as the orbitofrontal cortex (OFC), anterior cingulate (AC) gyrus, pre-supplementary and general motor areas (M1) may be involved in the encoding of long-known music and are later degenerating in the case of AD (Jacobsen et al., 2015). These regions of the brain, and regions within close proximity, also play fundamental roles in music-evoked emotion, theory of mind and sociability, connecting music with memories and share connectivity with deeper limbic areas such as the basal ganglia; a structure that is responsible not only for coordination of movement but also working memory and environmental conditioning (Hazy, Frank, \& O'Reilly, 2006; McNab \& Klingberg, 2008; Jacobsen et al., 2015; Wallmark, Deblieck, \& Iacoboni, 2018). Thus, like most stimuli, music has the ability to evoke recruitment of a vast array of neural regions; however, unlike many stimuli, music facilitates a multifunctional integration of regions spanning emotion, socialization, and in some instances for $\mathrm{PwD}$, even remembering.

The multimodal nature of music makes it a prime candidate for social interventions, as it allows people to engage in an emotionally and cognitively stimulating activity that facilitates both cognitive benefit as well as community engagement that serves to mitigate social stigma that accompanies dementia. Indeed, some of the misperceptions surrounding dementia include the notion that disease progression and corresponding cognitive decline is intertwined with loss of affect, self, and personhood. Music has the power to directly counter such misconceptions. Through music-based interventions, for example, individuals with dementia are exhibiting improvements in affect and social connectedness, sharing moments of joy and familiarity, and in some cases even promoting cognitive sparing as a consequence of mitigating stressors and increasing cognitive engagement (Blackburn \& Bradshaw, 
2014; Särkämö et al., 2016). Jane Austen's protagonist "Emma," from the novel of the same name, remarks that "without music, life would be blank to me" (Emma, 1815, p. 250), a sentiment that directly highlights how deeply personal, powerful, and life-affirming music can be. Thus, through social interventions in general, and music-based interventions in particular, it is becoming apparent that a diagnosis of dementia need not necessarily deny the enjoyment of those things which make us human.

\section{The Present Study: Voices in Motion}

The present study focused on implementing a social-cognitive intervention for persons with Alzheimer's and dementia and their caregivers, utilizing the benefits of music-based interventions. An overarching aim of Voices in Motion was to assess what impact an intergenerational choir group would have on psychological and cognitive function for those with dementia. The choir's structure was designed with particular attention to intergenerationality, music selection, and artistic direction in order to maximally engage all participants in the key social elements of the choir. The intergenerational component of the choir not only adds a youthfulness that our elderly demographic enjoys, but also offers an avenue for combating stigma through greater exposure and education of our high-school-aged adolescents. Music selection was predominately based upon popular songs of the past, especially songs relevant to our older demographic with dementia, as these are the songs most likely to be preserved in musical memory. Notably, our PwD were also tasked with remembering new songs, as previous findings have demonstrated that even those with dementia can learn new material given sufficient practice, expert direction, and compensatory mechanisms (e.g., relying upon procedural and emotional memory systems that are relatively spared; Baird \& Samson, 2015; Jacobsen et al., 2015). The musical repertoire was deliberately chosen to facilitate social connection as well as cognitive/physiological stimulation. In particular, we hypothesized that our choir would promote cognitive health, not through treating dementia itself, but rather by alleviating the common comorbidities of $\mathrm{AD}$ and dementia such as depression and social isolation. This hypothesis is consistent with the current literature, as it has been found that social isolation and depression are some of the most detrimental complications experienced by persons with dementia (Cipriani, Lucetti, Carlesi, Danti \& Nuti, 2015). By creating a supportive community environment which adds meaning and fosters the development of meaningful relationships, we expected depressive symptoms to decrease through 18 months of participation in this intergenerational choir. Additionally, despite the progression of the underlying disease process, we anticipated that any observed psychosocial improvements due to engagement in the choir would also result in the stabilization of cognitive function due to the lessened influence of these comorbidities.

\section{Method}

\section{Participants and Recruitment}

Participants were recruited through church choirs, doctors' offices, and local community centres. A phone interview was conducted to screen participants for eligibility. All participants were required to have mild to moderate dementia, as diagnosed by a physician, be willing to participate in research assessments, and have a caregiver who was also willing to participate in both the choir and research assessments. Recruitment was targeted at individuals with Alzheimer's Disease; however, all types of dementia were accepted. Two PwD had non-Alzheimer's dementia (frontal-temporal and vascular) and were included in analyses.

The University of Victoria's Institutional Review Board and the assisted-living facility director approved the study. Informed consent was obtained at the start of data collection for all participants. 
Participants were given a brief oral description of the study and then given a consent form to read and sign, with ample opportunity for questions. Choirs were composed of $\mathrm{PwD}$, their caregivers, and high-school students recruited from two participating schools. Students were awarded with community service hours. Participants were assigned to one of two choir groups and attended weekly two-hour choir practices consisting of a warm-up period, rehearsal, and thirty minutes of socialization. Song selection for each choir season was curated by a professional conductor based on the ages of the persons with dementia. While there were no official criteria for song selection, deliberate intent was focused on including well-known songs that evoked strong positive emotions (e.g., patriotism, collectiveness) and were famous during a critical period (i.e., from 13 to 30 years of age) in our elderly participants' lives. Choirs were active for three seasons, spanning 1.5 years. At the conclusion of each season, each choir performed a final concert that was open to the community.

Participants were recruited in two cohorts: cohort $1(\mathrm{n}=26)$ participated in all three choir seasons, while cohort $2(\mathrm{n}=28)$ participated in two seasons. Baseline assessments occurred at different times for each cohort. The mean age for $\mathrm{PwD}$ was 77.4 years ( $\mathrm{SD}=10.5 ; 75 \%$ female), and 67.1 years $(\mathrm{SD}=9.10)$ for caregivers ( $69 \%$ Female), $98.6 \%$ of all participants were Caucasian. The median MMSE score for PwD at intake was 24.0, which denotes mild dementia.

\section{Measures}

Throughout choir seasons, participants underwent numerous assessments, including routine social network analysis (SNA), focus groups, and a biopsychosocial (BPS) assessment battery, which included a neuropsychological assessment, cognitive tests, physical assessments, gait mapping, and a quality of life survey. A small subset of data was selected for the subsequent analyses in order to address the specified research questions mentioned above. Choir seasons two and three consisted of returning choir members (cohort 1) and newly recruited participants. The BPS battery was administered before choir participation, and approximately every four to six weeks during the choir season, as part of an intensive repeated measures design (Rast, MacDonald, \& Hofer, 2012; Stawski, MacDonald, Sliwinski, 2015). PwD and their caregivers underwent identical assessments individually, and were completed by trained student research assistants and physicians. Both caregivers and PwD vary widely in their experiences; the benefit of using this design is that it allows participants to serve as their own control (i.e., a within-person comparison), and to specifically gauge whether each individual exhibits improvement in function relative to person-specific initial levels for key outcomes (e.g., cognition, depressive symptoms). Such intensive micro longitudinal designs incorporate a baseline assessment (where pre-treatment levels of function are indexed) followed by numerous subsequent assessments that facilitate nuanced modelling of within-person change. Although a control group would allow for the exploration of between-group differences, the purpose of this research is not to examine the effectiveness of treatment (as is typical of intervention research); such between-group effects for music have already been reported elsewhere (see, for example, Särkämö et al., 2013). Rather, the present focus is to examine how social singing improves a given individual's symptomatic expression of dementia symptoms regardless of initial individual differences. Overall, the intensive repeated measures design is more powerful for examining the nuanced shape of change during a longitudinal study. Assessments entered a hiatus immediately following the season's final performance and resumed at the same intervals at the beginning of the following choir season.

The measures administered during the assessment battery spanned numerous functional domains, including physical health, global cognition, executive function, short-term memory, reaction time, emotional wellbeing, depression, mood, sleep, medications, socialization, spatial planning, quality of life, and activities of daily living, among others. As the data collected in this study span many facets and disciplines, analysis was limited to cognitive measures for $\mathrm{PwD}$ in order to focus on the 
current research questions.

\section{Cognitive measures.}

Measures of cognition included the Mini-mental State Examination (MMSE; Folstein, Folstein, \& McHugh, 1975), Trail Making Task A (TMTA; Lezak, 1995), and the Word Recall Task (Howard, 1980). The MMSE is a well-validated tool and measure of global cognition for PwD. It consists of verbally asking the participant questions about the world (e.g., What day is it?), orientation (e.g., What street do you live on?), memory, and perceptual-motor skills, and is scored out of 30. A score of 20-24 indicates mild cognitive impairment, $12-20$ is moderate, and less than 12 is severe cognitive impairment. The MMSE was selected as it is a standard measure in multi model neuropsychological batteries. TMTA is a timed task and a measure of perceptual processing speed. Participants were asked to draw a single line to connect 25 consecutive numbers without lifting the pen from the paper. Errors were corrected as needed, and error time was included in total task completion time. Recall indicates episodic memory ability. (e.g., types of birds, modes of transportation). Participants were given two minutes to memorize a list of 30 common English nouns. The nouns were categorizable into five distinct taxonomic categories (e.g., gemstones, modes of transportation, etc.). After two

minutes, participants were asked to recall as many of the words as possible, regardless of order. Score denotes total words remembered, with no penalty for false or repeated words.

\section{Psychosocial function.}

The Patient Health Questionnaire (PHQ-9) was used to index individual differences in the number of depressive symptoms and signs. The PHQ-9 was scored according to guidelines found in Kroenke and Spitzer (2002). Higher scores denoted a greater number of depressive symptoms and signs on a continuum, with scores of (a) 5-9 indicating minimal depression, (b) 10-14 reflecting minor depression, (c) 15-19 indicating major depression (moderately severe), and (d) 20+ reflecting major depression (severe).

\section{Statistical Procedure}

Linear mixed effect (multilevel) models were employed to index change for select indicators of cognitive and psychosocial function for persons with dementia $(\mathrm{PwD})$. Maximum likelihood estimation was used for all calculations, ensuring that all available data were analysed. Time was parameterized in terms of assessments, with time $=0$ reflecting initial baseline assessment.

\section{Results}

To explore the primary research objective, we employed linear mixed effect (multilevel) models index initial levels of select cognitive and psychosocial function at the outset of the choir, as well as the change in these measures for up to eight follow-up assessments spanning an 18-month period. Both level and change in function were modelled for global cognition (MMSE scores), perceptual processing speed (TMT-A completion time), episodic memory (Word Recall), as well as depressive symptoms and signs (PHQ-9 scores).

An initial model examined baseline level and long-term change in MMSE performance (see Figure 1). MMSE scores less than 24 are indicative of cognitive impairment, with expectations of continued decline for those with dementia. As shown in Figure 1, following the onset of the choir intervention at assessment 0, participants with dementia did not exhibit further significant decreases in MMSE scores spanning the up to an 18-month period. Although there was a wide range of MMSE scores 
$(2-28)$ at baseline, the median score was 24 . The population average slope for change in MMSE scores was 0.14 units per additional time of assessment $(\mathrm{p}=.381, \mathrm{CI}=-1.7$ to 2.7$)$.

A second model explored change in completion time for the TMT-A task - a putative measure of perceptual processing speed. Results demonstrated no significant change in performance for the TMT-A task during the course of choir participation (see Figure 2). At baseline, PwD exhibited a slight impairment to complete TMT-A, with an intercept of 79.7 seconds; based upon normative data, functional impairment is defined as having a completion time greater than 78 seconds (Gaudino, Geisler, \& Squires, 1995). For each additional point of assessment, participants' average TMT-A completion time slowed by an additional 5.79 seconds. This increase in completion time for TMT-A, however, was not significant $(\mathrm{p}=.39, \mathrm{CI}=-7.2-17.7)$ for persons with dementia; there was no inferential difference between baseline performance and performance up to 18 months later at follow-up 7.

A third multilevel model examined level of baseline function and post-choir-intervention change for the Word Recall task (see Figure 3). Persons with dementia were able to recall 5.55 words at baseline assessment (range $=0-22$ words). Further, a significant decline $(\mathrm{p}=.004, \mathrm{CI}=-3.8$ to -.76) of 0.33 words per additional point of assessment was observed. During the final assessment at follow-up 7, an average person with dementia recalled 3.25 of a possible 30 words. Scores ranged from 0 to 5 for participants during their final assessment.

A final multilevel model of change explored initial level and systematic change in depressive symptoms and signs over the course of the choir season as indexed by the PHQ-9. As demonstrated in Figure 4, results from this multilevel model indicated a significant decrease in depressive signs and symptoms by .48 points per additional assessment, $\mathrm{p}=.039$.

\section{Discussion}

The goal of this study was to investigate the potential benefits of engaging in an intergenerational choir on cognitive and psychosocial function for persons with dementia. $\mathrm{PwD}$ have shown an increased risk of developing depression and becoming socially isolated as the disease advances, with corresponding impacts on health (see, for example, Holt-Lunstad, Smith, Baker, Harris, \& Stephenson, 2015). These psychosocial health concerns, coupled with the ongoing neuropathological progression of $\mathrm{AD}$, are proposed to have compounding symptomatic effects (e.g., accelerated cognitive decline). For the Voices in Motion study, it was hypothesized that the social-cognitive choral intervention would act as a protective factor for cognition by alleviating co-existing depression and facilitating social connection for $\mathrm{PwD}$ and their caregivers.

The present findings demonstrated that $\mathrm{PwD}$ continued to show expected significant declines in episodic memory. As dementia is a progressive neurological disorder with no pharmacological treatment to slow disease course, the choir intervention was not expected to modulate episodic memory function that draws heavily upon damaged brain regions. Notably, PwD did not exhibit any significant losses for measures of general cognition or perceptual speed - two domains that exhibit early deficits during the dementia prodrome (see, for example, Backman, Jones, Berger, Laukka, \& Small, 2005) - as well as continued decline through the subsequent stages of dementia. Among the implications, the relative stability of the cognitive trends for MMSE and TMT-A suggest that the intergenerational choir intervention may be effective at preserving cognitive function for persons with dementia. The stability of the TMT-A suggests that, while impaired, areas associated with speed and executive functioning are not becoming further damaged. The stability of the MMSE score is particularly significant, as persons with dementia are known to have a mean decrease of 2.81 to 2.88 points per year (Salmon, Thal, Butters, \& Heindel, 1990; Roselli et al., 2009) and scores must decrease five points to be considered significant cognitive loss (Schmand et al., 1995). In our study, 
PwD did not show any losses on the MMSE over the 18 months of choir participation. Additionally, the reported number of depressive symptoms significantly decreased as a function of time spent in the choir.

Overall, these patterns suggest that the choir intervention confers a protective effect that can alleviate outcomes associated with underlying comorbidities and prolong cognitive health and patient independence, and could perhaps delay the transition into long term care. Potential explanations for choir's effectiveness include both the mitigation of depressive symptoms as well as the maintenance of social interactions, which are thought to promote positive affect, improved cortical functioning, better overall health, and reduced mortality risk (see, for example, Holt-Lunstad et al., 2015). Plausible mechanisms underlying our observed findings are discussed further in the ensuing sections.

\section{Why is Choir Effective? Some Plausible Mechanisms}

\section{Mitigating depressive symptoms.}

Depression is one of the most common comorbidities associated with dementia, with up to $63 \%$ of PwD affected (Burns, Jacoby, \& Levy, 1990). The effects of comorbid depression are well studied and detrimental to patient outcomes. Depression alone significantly reduces quality of life, and when combined with dementia, has compounding effects. For example, dementia patients with depression will transition to care homes faster than those with no depressive symptoms (Thomson et al., 2007). Indeed, caregivers often cite mood and behaviour changes as being the causal factor for transitioning $\mathrm{PwD}$ into care homes, with research suggesting these changes are accelerated due to depression (Kopez et al., 2000). Higher mortality rates for persons with comorbid depression and dementia has been well documented (Cuijers and Smit, 2002; Hoch et al., 1993; Perna et al., 2019).

The impact of depression on overall health, social connectedness, and cognitive function is well documented. In part, these negative effects reflect the deleterious impact that depression exerts on brain structures including the hippocampus and the prefrontal cortex (PFC) (Lebedeva et al., 2018). Depression is known to be associated with hypofrontality of the PFC, and, as a consequence of this, those with depression are shown to have poorer performance in tasks involving both episodic memory and executive functioning (Dillon \& Pizzagalli, 2018). Depression is also often related to chronic stress and heightened cortisol levels, the impacts of which can inhibit mesolimbic dopamine neurons, sensitize the amygdala's response to negative information, and suppress hippocampal neurogenesis - in the most severe cases, even causing prefrontal and limbic atrophy (Dillon \& Pizzagalli, 2018; Lebedeva et al., 2018). Depression and AD neurodegeneration exhibit damage to shared regions of the brain, and this overlap may explain why depression and AD produce compounded impairments on cognition (Thomson at al., 2007; Watkins and Brown, 2002).

By attempting to mitigate depressive symptomatology, it may be possible to improve cognitive functioning by limiting the impact of depression and other dementia-related comorbidities. Reducing the number of comorbidities for $\mathrm{PwD}$ may lead to fewer demands on available cognitive resources - a benefit that may be more apparent for cognitive abilities that do not draw directly upon brain regions most affected by $\mathrm{AD}$ progression. In the present study, this explanation may account for why episodic memory scores showed progressive decline across assessments despite choir participation, whereas performance stabilized for measures of general cognition and executive functioning. Social prescriptions (e.g., engaging in physical activity, arts-based programs) are now being employed in Canada and the UK to treat depression (Camic \& Chatterjee, 2013; Stickley and Hui, 2012); choir is an activity that may confer similar positive benefit. 
The Arbutus Review • $2019 \bullet$ Vol. 10, No. 1 • https://doi.org/10.18357/tar101201918962

\section{Avoiding social isolation.}

Social isolation is another common complication for $\mathrm{PwD}$, which, like depression, increases the likelihood of negative outcomes such as decreased survival rates and negative affect (Friedler, Crapser, and McCullough, 2015; Mehta, Yaffe, Langa, Sands, Whooley, \& Covinsky, 2003). Social isolation and loneliness are known to impair health by raising levels of stress hormones (e.g., cortisol) and inflammation, and such elevations, in turn, can increase the risk of heart disease, arthritis, Type 2 diabetes, and dementia (Holt-Lunstad et al., 2015). It is not uncommon for PwD to socially withdraw from their communities, thereby reducing their support network and increasing their risk of ailments associated with social isolation (Van der Wee et al., 2019). The cognitive impacts of social isolation in $\mathrm{PwD}$ are similar to those seen in depression, with participants often experiencing decreased cognitive functioning, executive functioning, and episodic memory (Mehta et al., 2013). Therefore, performance deficits for $\mathrm{PwD}$ would be more pronounced for those who have existing comorbidities, such as depression or social isolation.

Several hypotheses have attempted to explain the protective effects of social relationships on overall health. The buffering hypothesis, for example, posits that positive relationships provide resources (informational, emotional, or tangible) that promote adaptive behavioural and neurological responses to acute or chronic stressors (Holt-Lunstad et al., 2010). Relational quality is determined predominantly by three contributing factors: the amount of integration in a social network, the intended support within a social network, and the perception or belief by the individual that they are supported within a social network (Holt-Lunstad et al., 2010). By facilitating an intergenerational choir of relational peers, youth, and thoughtful volunteers, the Voices in Motion study aims to foster a network that achieves these criteria. Accordingly, in the present study, a potential explanation for the observed reductions in depressive symptoms and stability in cognitive functioning may reflect the buffering effect that social relationships have against psychosocial stressors.

\section{Creating an intergenerational social milieu.}

Relative to singing alone or isolated music therapy, social (choral) singing is thought to be more effective at mitigating depression and social isolation (Chang et al., 2015). By creating a choral milieu that includes other PwD, caregivers, and high school students, the Voices in Motion study effectively engaged multiple generations and created a supportive community where all members worked towards a common goal: to participate in and enjoy music. Music has been shown to reduce depressive symptoms in PwD (Blackburn \& Bradshaw, 2014; Change et al., 2015); by further combining a music program with an intergenerational component, Voices in Motion also fostered the development of meaningful social relationships. The goal was to directly counter the tendency for $\mathrm{PwD}$ to socially withdraw, as well as to buffer the effects of loneliness and the stigma of dementia.

Based upon qualitative reports from focus groups, the intergenerational component was said to add significant value to both the community and the experience, perhaps best summarized as a quote from one participant: "I just love seeing the kids here. We live in a senior complex with too many old people. All our friends are old, my husband is old. I'm just so ... sick of old people." Music was the catalyst by which an intergenerational community was formed, and this community proved to mitigate depressive symptoms (significant reductions in depressive symptoms and signs were observed), social isolation (social network analysis indicated a richness to social interactions by the conclusion of the choir season), and stigma (the end of year intergenerational concert served to counter stereotypes regarding what $\mathrm{PwD}$ can achieve). 
The Arbutus Review • $2019 \bullet$ Vol. 10, No. 1 • https://doi.org/10.18357/tar101201918962

\section{Integrating multiple memory systems.}

The progression of neuropathology for those with Alzheimer's Disease is well characterized, with cognitive-behavioural deficits (e.g., early changes in memory function) presenting themselves in a predictable manner (see, for example, Aisen et al., 2017; Braak \& Braak, 1991). Biochemical protein changes in the entorhinal cortex are typical of the early stages of $\mathrm{AD}$, with subsequent progression gradually degenerating tissue in the peripheral cortices. Despite changes to specific brain structures for those with $\mathrm{AD}$, many regions of the brain are spared until very late stages, particularly those regions implicated in musical memory (Clark \& Warren, 2015; Jacobsen et al., 2015; Peck et al., 2016). The power of music for enhancing mood and wellbeing is well documented; social singing in a choir also elicits neurochemical brain changes that enhance "contact, coordination, and cooperation" with others (Keeler et al., 2015). Even those with advanced Alzheimer's Disease benefit from singing overlearned songs from the past, as social singing relies less upon damaged brain regions linked to memory (e.g., the hippocampus), and more upon emotional (limbic) and procedural (striatal) brain systems. Due to the later AD-related neurodegeneration of specific cortices, elements of music-evoked emotion, musical knowledge and competency, and even musical memory can show relative sparing. The specific cortices related to such abilities include the orbitofrontal cortex (OFC), anterior cingulate gyrus, and supplementary and general motor areas, all of which are share extensive connections to deeper limbic systems.

Regions of the brain associated with procedural memory (e.g., the striatum), which is responsible for the training and automaticity of certain tasks, are also well preserved despite the progression of $\mathrm{AD}$. As music draws upon this system, $\mathrm{PwD}$ can still fluidly play musical instruments and sing overlearned songs despite severely impaired memory and cognition. Persons with AD also show a spared ability of memory to recognize pieces of music based on melody or lyrics (Clark \& Warren, 2015). This preserved ability reflects the involvement of another preserved system - semantic memory - linked to heightened sympathetic arousal in the presence of familiar songs or stimuli. This semantic system is also responsible, in part, for the ability of $\mathrm{PwD}$ to learn new songs over the course of vigorous practice (Baird \& Samson, 2015). However, a key feature of this system is that the $\mathrm{PwD}$ cannot typically recall where they know the song from or when they heard it last, only that it is familiar and they can engage with it. Overall, the potential compensatory strengths of music in general and choir, in particular, are seemingly obvious: music is truly a superstimulus that integrates multiple memory systems, permitting those with dementia to participate in choir to the same degree as any other chorister.

\section{Limitations}

In terms of research design and assessment, this study is among the most ambitious investigations of the effects of an intergenerational choir on persons with dementia. However, several limitations of the present study should be noted. First, due to the size and scope of the battery, participants may have become fatigued during assessments, with a corresponding deleterious influence on performance. We attempted to mitigate this concern by block randomizing the order of task administration across assessments, and by assigning select tasks (e.g., health, sleep quality) as take-home assessments from the beginning of the second choir season. A second notable limitation concerns sample size. The advanced quantitative models of change and corresponding estimators (maximum likelihood) employed for this study necessitate sufficient sample size. Although the total number of participants was modest for conducting such analyses of change, the intensive repeated measures research design served to offset this concern. A final limitation worthy of mention concerns practice effects. Repeated exposure to the same testing instruments, particularly with short retest intervals, may serve to mask the true rate of change in performance for various outcome measures. In part, this issue 
was addressed in the present study through the use of alternate forms for tasks susceptible to practice (e.g., numerous alternate forms of the episodic memory word lists) as well as the inclusion of measures known to be relatively impervious to practice effects (e.g., perceptual processing speed).

\section{Implications and Future Directions}

The results of the current study suggest that an intergenerational, music-based intervention can be successful at preserving general cognition and executive functioning during dementia-related memory loss. This is not only obviously beneficial for $\mathrm{PwD}$, but is also extremely valuable for their caregivers and the healthcare system in general. The advent of choral participation for PwD and their caregivers has been shown to facilitate stronger relationships between one another (Grocke, 2012), to promote positive affect in the caregivers (Han \& Radel, 2016), and to foster improved general health and well-being for both parties (Grocke, 2012; Holt-Lunstad et al., 2010). Benefits of such non-pharmacological interventions for the healthcare system include preserving the health of caregivers, improving the quality of life for $\mathrm{PwD}$, and considerable healthcare savings by delaying the transition of PwD to subsequent levels of long-term care. Federal spending on dementia care is currently $\$ 15$ billion and is projected to increase substantially over the next several decades as the population ages and incidence of dementia increases. Additionally, pharmaceutical non-compliance rates are high among the elderly, particularly in rural communities (Grewal, et al., 2108). Whereas medication adherence rates in $\mathrm{PwD}$ are not well documented, having a non-pharmaceutical option to mitigate dementia-related symptoms would serve to augment care options (Arlt, Linder, Rosler, \& von Renteln-Kruse, 2008; Stewart, Jameson, \& Curtin, 2015). It has also been suggested that self-stigma and perceived community standards are related to attitudes towards mental health and mental health treatment in older adults. Developing a non-pharmacological treatment for depression in $\mathrm{PwD}$ can provide patients more agency in their care and reduce the negative outcomes associated with coexisting depression, including keeping adults in their homes longer and delaying the transition to long-term care. A choir intervention would be relatively simple and cost effective to place in a rural setting and may also serve to help reduce stigma in the community.

Further investigation is required to determine the full potential of choir as a socially-prescribed lifestyle intervention for those with dementia. This study collected hundreds of measures of physical, cognitive, psychological, and social health for all participants. Only a small subset of that data was used to address global cognition, processing speed, episodic memory, and depression as a function of participation in the intervention. All collected data is intended for future use to fully analyse the biopsychosocial benefits of a community choir. In particular, a key focus of future research must also evaluate the potential benefit of intergenerational choirs on caregivers. Caregivers for PwD can often experience burnout, negative affect and increased levels of distress and chronic illness (Vitaliano, Young, \& Zhang, 2004). These issues, compounded with the deteriorating health of the $\mathrm{PwD}$, can cause a faster transition to long-term care for those with dementia. Notably, it has been substantiated in previous choral studies that caregivers will show reductions in stress and depression, and increases in positive affect both in response to seeing their $\mathrm{PwD}$ engage joyfully in social activities and in forming new positive relationships with peers in similar circumstances (see, for example, Han \& Radel, 2016). Additionally, the choir aims to expand from solely community-based choirs into residential care facilities. Participants in care facilities are expected to show similar results to those in the community-based program; however, as many of those living in residential care have a pre-established community, the benefits of choir in this context remain unknown. Another potential line of research involves incorporating neuroimaging to assess the functional and structural changes that accompany the changes in behavioural and cognitive performance. For example, functional near-infrared spectroscopy (fNIRS) could be employed to assess the neural correlates of social 
singing. Novel insights provided through such fNIRS investigations could identify the extent to which music-based interventions stimulate functional adaptations in the brain that directly promote cognitive-behavioural health for those with dementia. 
The Arbutus Review • $2019 \bullet$ Vol. 10, No. 1 • https://doi.org/10.18357/tar101201918962

\section{References}

Aisen, P. S., Cummings, J., Jack, C. R., Morris, J. C., Sperling, R., Frölich, L., ... \& Scheltens, P. (2017). On the path to 2025: understanding the Alzheimer's disease continuum. Alzheimer's Research \& Therapy, 9(1), 60-70. https://doi.org/10.1186/s13195-017-0283-5

Arlt, S., Lindner, R., Rösler, A., \& von Renteln-Kruse, W. (2008). Adherence to medication in patients with dementia. Drugs \& Aging, 25 (12), 1033-1047. https://doi.org/10.2165/0002512200825120-00005]

Austen, J. (1815). Emma. New York, NY: Penguin Books.

Bäckman, L., Jones, S., Berger, A. K., Laukka, E. J., \& Small, B. J. (2005). Cognitive impairment in preclinical Alzheimer's disease: A meta-analysis. Neuropsychology, 19(4), 520-531. http://dx.doi.org/10.1037/0894-4105.19.4.520

Baird, A., \& Samson, S. (2009). Memory for music in Alzheimer's disease: unforgettable?. Neuropsychology Review, 19(1), 85-101. https://doi.org/10.1007/s11065-009-9085-2

Baird, A., \& Samson, S. (2015). Music and dementia. Progress in Brain Research, 217, 207-235. https://doi.org/10.1016/bs.pbr.2014.11.028

Bannan, N., \& Montgomery-Smith, C. (2008). "Singing for the brain": Reflections on the human capacity for music arising from a pilot study of group singing with Alzheimer's patients. Journal of the Royal Society for the Promotion of Health, 128(2), 73-78. https://doi.org/10.1177/1466 424007087807

Behuniak, S. M. (2011). The living dead? The construction of people with Alzheimer's disease as zombies. Ageing \& Society, 31(1), 70-92. https://doi.org/10.1017/S0144686X10000693

Beynon, C. (2017). Never too old: Establishing an intergenerational choir for transformational learning through singing. The Choral Journal, 57(7), 18-29.

Beynon, C., \& Lang, J. (2018). The more we get together, The more we learn: Focus on intergenerational and collaborative learning through singing. Journal of Intergenerational Relationships, 16(1-2), 45-63. https://doi.org/10.1080/15350770.2018.1404405

Blackburn, R., \& Bradshaw, T. (2014). Music therapy for service users with dementia: A critical review of the literature. Journal of Psychiatric and Mental Health Nursing, 21(10), 879-888. https://doi.org/10.1111/jpm.12165

Braak, H., \& Braak, E. (1991). Neuropathological stageing of Alzheimer-related changes. Acta Neuropathologica, 82(4), 239-259. https://doi.org/10.1007/BF00308809

Burns, A., Jacoby, R., \& Levy, R. (1990). Psychiatric phenomena in Alzheimer's Disease. III: Disorders of mood. The British Journal of Psychiatry, 157(1), 81-86. https://doi.org/10.1192/bjp. 157.1.81

Camic, P. M., \& Chatterjee, H. J. (2013). Museums and art galleries as partners for public health interventions. Perspectives in Public Health, 133(1), 66-71. https://doi.org/10.1177/175791391246 8523

Chang, Y. S., Chu, H., Yang, C. Y., Tsai, J. C., Chung, M. H., Liao, Y. M., .. \& \& Chou, K. R. (2015). The efficacy of music therapy for people with dementia: A meta-analysis of randomised controlled trials. Journal of Clinical Nursing, 24(23-24), 3425-3440. https://doi.org/10.1111/ jocn.12976

Cipriani, G., Lucetti, C., Carlesi, C., Danti, S., \& Nuti, A. (2015). Depression and dementia. A review. European Geriatric Medicine, 6(5), 479-486. https://doi.org/10.1016/j.eurger.2015.07.010

Clark, C. N., \& Warren, J. D. (2015). Music, memory and mechanisms in Alzheimer's disease. Brain, 138(8), 2122-2125. https://doi.org/10.1093/brain/awv148

Cohen, ES. (2001). The complex nature of ageism: What is it? Who does it? Who perceived it? Gerontologist, 41(5), 576-577. http://dx.doi.org/10.1093/geront/41.5.576 
Cuijpers, P., \& Smit, F. (2002). Excess mortality in depression: A meta-analysis of community studies. Journal of Affective Disorders, 72(3), 227-236. https://doi.org/10.1016/S01650327(01)00413-X

Cummings, J. (2012). Alzheimer's disease diagnostic criteria: Practical applications. Alzheimer's Research 83 Therapy, 4 (4), 35-41. https://doi.org/10.1186/alzrt138

Grocke D. (2012). Connecting through music: A study of a spousal caregiver-directed music intervention designed to prolong fulfilling relationships in couples where one person has dementia/A response to Felicity Baker, Denise Grocke and Nancy Pachana's article. The Australian Journal of Music Therapy, 23, 4-21.

Dillon, D. G., \& Pizzagalli, D. A. (2018). Mechanisms of memory disruption in depression. Trends in Neurosciences, 41(3), 137-149. https://doi.org/10.1016/j.tins.2017.12.006

Dodd, D. (2018, March 12). How to harness music to fight dementia. Retrieved from https://www.ft. com/content/62f912b0-100a-11e8-8cb6-b9ccc4c4dbbb

Friedler, B., Crapser, J., \& McCullough, L. (2015). One is the deadliest number: The detrimental effects of social isolation on cerebrovascular diseases and cognition. Acta Neuropathologica, 129(4), 493-509. https://doi.org/10.1007/s00401-014-1377-9

Folstein, M. F., Folstein, S. E., \& McHugh, P. R. (1975). "Mini-mental state": A practical method for grading the cognitive state of patients for the clinician. Journal of Psychiatric Research, 12(3), 189-198.

Gaudino, E. A., Geisler, M. W., \& Squires, N. K. (1995). Construct validity in the Trail Making Test: What makes Part B harder? Journal of Clinical and Experimental Neuropsychology, 17(4), 529-535. https://doi.org/10.1080/01688639508405143

Glazner, G., \& Kaplan, D. B. (2018). The Alzheimer's Poetry Project. Jama, 320(22), 2294-2295. https://doi.org/10.1001/jama.2018.16340

Grewal, Sheets, D., Smith, A.P., Trites, M., Kennedy, M., MacDonald, S.W.S. (2018, May). A Community Choir to Facilitate Psychosocial and Cognitive Health for Caregivers and Persons with Dementia. Poster session presented at Rural Dementia Action Research Summit 2018.

Han, A., \& Radel, J. (2016). Spousal caregiver perspectives on a person-centered social program for partners with dementia. American Journal of Alzheimer's Disease 63 Other Dementias, 31 (6), 465-473. https://doi.org/10.1177/1533317515619036

Harris, P. B., \& Caporella, C. A. (2014). An intergenerational choir formed to lessen Alzheimer's disease stigma in college students and decrease the social isolation of people with Alzheimer's disease and their family members: A pilot study. American Journal of Alzheimer's Disease 8 Other Dementias, 29(3), 270-281.

Hazy, T. E., Frank, M. J., \& O'Reilly, R. C. (2006). Banishing the homunculus: making working memory work. Neuroscience, 139(1), 105-118. https://doi.org/10.1177/1533317513517044

Hoch, C. C., Reynolds III, C. F., Buysse, D. J., Fasiczka, A. L., Houck, P. R., Mazumdar, S., \& Kupfer, D. J. (1993). Two-year survival in patients with mixed symptoms of depression and cognitive impairment: comparison with major depression and primary degenerative dementia. The American Journal of Geriatric Psychiatry, 1(1), 59-66. https://doi.org/10.1097/00019442199300110-00008

Holt-Lunstad, J., Smith, T. B., \& Layton, J. B. (2010). Social relationships and mortality risk: a metaanalytic review. PLoS medicine, 7(7), 1-20. https://doi.org/10.1371/journal.pmed.1000316

Holt-Lunstad, J., Smith, T. B., Baker, M., Harris, T., \& Stephenson, D. (2015). Loneliness and social isolation as risk factors for mortality: a meta-analytic review. Perspectives on Psychological Science, 10(2), 227-237. https://doi.org/10.1177/1745691614568352

Howard, D. V. (1980). Category norms: A comparison of the Battig and Montague (1969) norms with the responses of adults between the ages of 20 and 80. Journal of Gerontology, 35(2), 
The Arbutus Review • $2019 \bullet$ Vol. 10, No. 1 • https://doi.org/10.18357/tar101201918962

225-231. https://doi.org/10.1093/geronj/35.2.225

Hsiao, Y. H., Chang, C. H., \& Gean, P. W. (2018). Impact of social relationships on Alzheimer's memory impairment: mechanistic studies. Journal of Biomedical Science, 25(1), 3. https://doi.org/ 10.1186/s12929-018-0404-x

Jacobsen, J. H., Stelzer, J., Fritz, T. H., Chételat, G., La Joie, R., \& Turner, R. (2015). Why musical memory can be preserved in advanced Alzheimer's disease. Brain, 138(8), 2438-2450. https://doi.org/10.1093/brain/awv135

Kopetz, S., Steele, C. D., Brandt, J., Baker, A., Kronberg, M., Galik, E., ...\& Lyketsos, C. G. (2000). Characteristics and outcomes of dementia residents in an assisted living facility. International Journal of Geriatric Psychiatry, 15(7), 586-593. https://doi.org/10.1002/10991166(200007)15:7<586::AID-GPS148>3.0.CO;2-D

Lebedeva, A., Sundström, A., Lindgren, L., Stomby, A., Aarsland, D., Westman, E., ...\& Nyberg, L. (2018). Longitudinal relationships among depressive symptoms, cortisol, and brain atrophy in the neocortex and the hippocampus. Acta Psychiatrica Scandinavica, 137(6), 491-502. https://doi.org/10.1111/acps.12860

Lezak, M. D. (1995). Neuropsychological Assessment (3rd ed.). New York, NY: Oxford University Press.

Mehta, K. M., Yaffe, K., Langa, K. M., Sands, L., Whooley, M. A., \& Covinsky, K. E. (2003). Additive effects of cognitive function and depressive symptoms on mortality in elderly community-living adults. The Journals of Gerontology Series A: Biological Sciences and Medical Sciences, 58(5), M461-M467. https://doi.org/10.1093/gerona/58.5.M461

McNab, F., \& Klingberg, T. (2008). Prefrontal cortex and basal ganglia control access to working memory. Nature Neuroscience, 11 (1), 103-107. https://doi.org/10.1038/nn2024

Mineo, L. (2017, April 11). Over nearly 80 years, Harvard study has been showing how to live a healthy and happy life. Retrieved from/https://news.harvard.edu/gazette/story/2017/04/overnearly-80-years-harvard-study-has-been-showing-how-to-live-a-healthy-and-happy-life/

Palmore, E.B. (1999). Ageism: Negative and Positive. New York, NY: Springer.

Perna, L., Wahl, H. W., Weberpals, J., Jansen, L., Mons, U., Schöttker, B., \& Brenner, H. (2019). Incident depression and mortality among people with different types of dementia: results from a longitudinal cohort study. Social Psychiatry and Psychiatric Epidemiology, 54(7), 1-9. https://doi.org/10.1007/s00127-019-01683-0

Petersen, R. C. (2004). Mild cognitive impairment as a diagnostic entity. Journal of Internal Medicine, 256(3), 183-194. https://doi.org/10.1111/j.1365-2796.2004.01388.x

Rast, P., MacDonald, S. W., \& Hofer, S. M. (2012). Intensive measurement designs for research on aging. GeroPsych, 25, 45-55. https://doi.org/10.1024/1662-9647/a000054

Roselli, F., Tartaglione, B., Federico, F., Lepore, V., Defazio, G., \& Livrea, P. (2009). Rate of MMSE score change in Alzheimer's disease: Influence of education and vascular risk factors. Clinical Neurology and Neurosurgery, 111(4), 327-330. https://doi.org/10.1016/j.clineuro.2008.10.006

Salmon, D. P., Thal, L. J., Butters, N., \& Heindel, W. C. (1990). Longitudinal evaluation of dementia of the Alzheimer type: A comparison of 3 standardized mental status examinations. Neurology, 40(8), 1225-1225. https://doi.org/10.1212/WNL.40.8.1225

Särkämö, T., Laitinen, S., Numminen, A., Kurki, M., Johnson, J. K., \& Rantanen, P. (2016). Clinical and demographic factors associated with the cognitive and emotional efficacy of regular musical activities in dementia. Journal of Alzheimer's Disease, 49(3), 767-781. https://doi.org/10.3233/JAD-150453

Schmand, B., Lindeboom, J., Launer, L., Dinkgreve, M., Hooijer, C., \& Jonker, C. (1995). What is a significant score change on the mini-mental state examination? International Journal of Geriatric Psychiatry, 10(5), 411-414. https://doi.org/10.1002/gps.930100510 
Simmons-Stern, N. R., Budson, A. E., \& Ally, B. A. (2010). Music as a memory enhancer in patients with Alzheimer's disease. Neuropsychologia, 48(10), 3164-3167. https://doi.org/10.1016/j.neu ropsychologia.2010.04.033

Stawski, R. S., MacDonald, S. W., \& Sliwinski, M. J. (2015). Measurement burst design. The Encyclopedia of Adulthood and Aging, 1-5. https://doi.org/10.1002/9781118521373.wbeaa313

Stewart, H., Jameson, J. P., \& Curtin, L. (2015). The relationship between stigma and self-reported willingness to use mental health services among rural and urban older adults. Psychological Services, 12(2), 141-148. http://dx.doi.org/10.1037/a0038651

Stickley, T., \& Hui, A. (2012). Social prescribing through arts on prescription in a UK city: participants' perspectives (part 1). Public Health, 126(7), 574-579. https://doi.org/10.1016/j.puhe. 2012.04 .002

Squire, L.R. (2009). Memory and Brain Systems: 1969-2009. Journal of Neuroscience, 29, 12711-12716. https://doi.org/10.1523/JNEUROSCI.3575-09.2009

Thompson, S., Herrmann, N., Rapoport, M. J., \& Lanctôt, K. L. (2007). Efficacy and safety of antidepressants for treatment of depression in Alzheimer's Disease: A metaanalysis. The Canadian Journal of Psychiatry, 52(4), 248-255. https://doi.org/10.1177/070674370705200407

Van der Wee, N. J., Bilderbeck, A. C., Cabello, M., Ayuso-Mateos, J. L., Saris, I. M., Giltay, E. J., ... \& Porcelli, S. (2019). Working definitions, subjective and objective assessments and experimental paradigms in a study exploring social withdrawal in schizophrenia and Alzheimer's disease. Neuroscience \&5 Biobehavioral Reviews, 97, 38-46. https://doi.org/10.1016/j.neubiorev. 2018.06.020

Vitaliano, P. P., Young, H. M., \& Zhang, J. (2004). Is caregiving a risk factor for illness?. Current Directions in Psychological Science, 13(1), 13-16. https://doi.org/10.1111/j.09637214.2004.01301004.x

Wallmark, Z., Deblieck, C., \& Iacoboni, M. (2018). Neurophysiological effects of trait empathy in music listening. Frontiers in Behavioral Neuroscience, 12, 66. https://doi.org/10.3389/fnbeh.2018. 00066

WHO. (2019, Sept. 19). Dementia. Retrieved from https://www.who.int/news-room/fact-sheets/de tail/dementia 